\title{
Acute Toxicity in Nasopharyngeal Carcinoma Patients Treated with IMRT/VMAT
}

\author{
Sevim Ozdemir*, Mustafa Akin, Yasin Coban, Cumhur Yildirim, Omer Uzel
}

\begin{abstract}
Purpose: To evaluate acute toxicity in nasopharyngeal cancer (NPC) patients treated with intensity modulated radiotherapy (IMRT)/volumetric modulated arc therapy (VMAT) with or without cisplatin-based chemotherapy. Materials and Methods: A total of 45 newly diagnosed, histologically proven non-metastatic NPC patients treated with IMRT between May 2010 and December 2012, were evaluated retrospectively, 37 planned with Eclipse and 8 with Prowess Panther treatment planning system. The doses to the planning target volumes of primary tumor and involved lymph nodes, high risk region, and uninvolved regional nodal areas were $70 \mathrm{~Gy}, 60 \mathrm{~Gy}$, and $54 \mathrm{~Gy}$ respectively and delivered simultaneously over 33 fractions to 39 patients. Another 6 patients irradiated with sequential boost technique. Some $84.4 \%$ of patients received chemotherapy. Acute toxicities were graded according to the Radiation Therapy Oncology Group scoring criteria and Common Terminology Criteria for Adverse Events (CTCAE) for chemotherapy side effects. Results: Median age was 43 years (14-79) and all patients were WHO type II. Grade 1 mucositis and dysphagia were observed in $17(37.8 \%)$, and $10(22.2 \%)$ patients, respectively. The incidence of acute grade 2 mucositis and dysphagia was $55.6 \%$ and $68.9 \%$, respectively. The most common chemoradiotherapy related acute toxicities were nausea, leucopenia and thrombocytopenia. Grade 3 toxicity was detected in $13(28.8 \%)$ cases. No grade 4 toxicity was occurred. Mean weight loss was $9 \%$. None of the patients required the insertion of percutaneous endoscopic gastrostomy for nutritional support. Radiation therapy was completed without interruption in all patients. Conclusions: IMRT is a safe and effective treatment modality, and well tolerated by patients in the treatment of nasopharyngeal carcinoma. No unexpected side effects were observed.
\end{abstract}

Asian Pac J Cancer Prev, 16 (5), 1897-1900

\section{Introduction}

Nasopharyngeal carcinoma (NPC) frequency varies according to ethnic groups and geographic regions, being common in the southeast Asia and southern China. NPC has a special place among head and neck cancers due to epidemiological and histological characteristics and complex geometry. Whereas NPC is sensitive to radiotherapy (RT) and chemotherapy (CT). RT is the standard treatment in non-metastatic NPC. High survival rates are achieved with RT alone in early stage lesions while concomitant chemoradiotherapy (CRT) is the standard approach for locally advanced tumors.

The improvement in tumor target coverage and significant sparing of adjacent critical structures allow the feasibility of intensity modulated radiotherapy (IMRT) in NPC (Lee et al., 2002; Phua Chee Ee et al., 2013). The superiority of IMRT in the treatment of NPC in terms of local control and treatment-related toxicity has been shown in several studies (Lee et al., 2005; Leung et al., 2005; Chen et al., 2008). However, two points should be considered in the application of this technique. First; to care organ motion in the treatment field and set-up errors, second; to provide implementation of planning and delivered dose accurately to the correct target. Therefore, several IGRT (image guided radiotherapy) applications have emerged. If IMRT done with IGRT high therapeutic doses can be reached with acceptable toxicity.

Despite all these; temporary toxicities has been shown with IMRT. While grade 3 and higher mucositis has been reported $65 \%$ with conventional techniques it was about 23-44\% after IMRT with chemotherapy (Wong et al., 2010; Wang et al., 2013; Sun et al., 2014). Besides, grade 3 and higher dysphagia has been reported as $32 \%$ with IMRT (Saleh-Ebrahimi et al., 2013). Studies demonstrated that total incidence of grade 3 or 4 acute toxicities in patients receiving concurrent chemotherapy was higher than those who received IMRT alone (Lee et al., 2005; Wong et al., 2010; Saleh-Ebrahimi et al., 2013; Wang et al., 2013; Sun et al., 2014).

This study aimed to evaluate acute toxicity in NPC patients treated with IMRT/VMAT with or without cisplatin-based CT.

\section{Materials and Methods}

\section{Patients' characteristics}

A total of 45 newly diagnosed, histologically 
proven non-metastatic NPC patients treated with IMRT between May 2010 and December 2012, were evaluated retrospectively. The routine workup included; medical history, physical and fiberoptic endoscopic examination of the nasopharynx, complete blood count, serum chemistry panel, magnetic resonance imaging (MRI) of the head and neck and PET-CT were done before initiation of RT. Patients were classified according to American Joint Committee on Cancer (AJCC) staging system 2009 (Edge et al., 2009).

\section{Radiotherapy planning}

Immobilization of patients was provided with thermoplastic head and shoulder mask. Imaging was performed from the top of the head to the lower part of the sternoclavicular joint with $2.5 \mathrm{~mm}$ sliced images. PET-CT and MRI images were fusioned with planning CT for all patients. 37 patients were planned by using the Eclipse (ver. 8.6) treatment planning system (19 patients were planned with VMAT-18 patients were planned with dynamic IMRT). Eight cases were planned by using the planning system of Prowess Panther V5.01.

The gross tumor volume (GTV70) is defined as primary tumor and involved lymph nodes considering physical examination, endoscopic findings, CT, PET-CT, and magnetic resonance imaging (MRI). The clinical target volumes (CTVs) were created as; CTV70: GTV+ 5mm margin, CTV60; entire nasopharynx. CTV54 defined as low risk region (entire nasopharynx, posterior ethmoids, posterior third of nasal cavity and maxillary sinuses, inferior sphenoid sinus, clivus, cavernous sinuses and elective nodal areas). While neck lymph node level II-V were included in CTV54 in all cases, level Ib was included when an adjacent level is involved. PTV was created by adding $3 \mathrm{~mm}$ margin to CTV. The eyes, lenses, optic nerves, chiasm, pituitary gland, mandible, temporal lobes, brain stem, spinal cord, parotid glands, submandibular glands, oral cavity, temporomandibular joints, larynx, thyroid gland, cochleas, pharynegeal muscles and the brachial plexus were delineated as organs at risk. Target volumes and critical organs were delineated according to RTOG atlas.

The doses to the planning target volumes of primary tumor and involved lymph nodes, high risk region, and uninvolved regional nodal areas were $70 \mathrm{~Gy}, 60 \mathrm{~Gy}$, and 54 Gy respectively and delivered simultaneously over 33 fractions to 39 patients. And other 6 patients received 70 Gy to primary tumor and involved lymph nodes and 50 Gy for electively irradiated neck nodes with sequential boost tecnique.

Planning objectives: The treatment goals were; at least $95 \%$ of the PTV volume would receive $100 \%$ of determined dose, and the maximum dose (Dmax) would not exceed $107 \% .98 \%$ of PTV70 volume should receive $95 \%$ of prescribed dose. The volume of PTV received more than $107 \%$ of the prescription dose should not exceed $2 \%$. For OARs dose constraints from the RTOG were taken as reference. According to this, maximum doses to spinal cord and brain stem were limited to $45 \mathrm{~Gy}$ and 54 Gy respectively. At least one side of the parotid gland mean dose was aimed to be less than 26 Gy or the volume receiving $30 \mathrm{~Gy}$ radiation should be less than $50 \%$ of parotid volume.

\section{Treatment delivery}

Patients who were planned with Eclipse planning system underwent on-board kV-CBCT imaging (Varian On-Board Imaging version 1.5, Varian Medical Systems, Palo Alto, CA) during each fraction of treatment. These images were fused with the planning CT images. Alignments were based on both clivus and spinal cord. Manual adjustment was done if necessary. Treatment was delivered with Varian Rapid-Arc lineer accelerator. The patients who were planned with Prowess Panther V5.01 planning system; treatment was delivered on an Elekta Synergy Linac with step-and-shoot IMRT. kV portal imaging was registered with planning images based on both clivus and spinal cord.

\section{Chemotherapy}

Thirty eight (84.4\%) patients received chemotherapy. The regimen of neoadjuvant CT included 3 cycles of TPF (docetaxel $75 \mathrm{mg} / \mathrm{m}^{2} /$ day, day 1 ; cisplatin $75 \mathrm{mg} /$ $\mathrm{m}^{2} /$ day, day 1 ; 5-fluorouracil $750 \mathrm{mg} / \mathrm{m}^{2} /$ day, days $1-5$ ) and administered to $15(33 \%)$ patients every 3 weeks. Concurrent cisplatin CT $\left(100 \mathrm{mg} / \mathrm{m}^{2}\right)$, was administered to 38 patients $(84.4 \%)$ on the first, twenty second and forty third days during treatment.

\section{Follow up}

During radiotherapy, all patients were observed and toxicity form was filled out per week in order to evaluate the acute side effects. Following completion of all therapy, patients were fully evaluated at 1 . and 3 . month. Acute toxicities (mucositis, dysphagia, hematologic toxicity vb.) were graded according to the Radiation Therapy Oncology Group (RTOG) scoring criteria and CTCAE for chemotherapy side effects.

\section{Statistical analysis}

Descriptive statistical methods were used to examine study data.

\section{Results}

\section{Patients' characteristics}

Median age was 43 years (14-79) and $73 \%$ of the patients were male. All patients were WHO type II, undifferentiated type was the predominant histology in our study group. The majority of patients showed advanced clinical stages. Karnofsky Performance Status Scale was 70-90\% for all patients. Patients' characteristics and treatment details are summarized in Table 1.

\section{Treatment toxicity}

Grade 1 mucositis and dysphagia were observed in 17 (\%37.8), and 10 (\%22.2) patients, respectively. The incidence of acute grade 2 mucositis and dysphagia was \%55.6 and \%68.9, respectively. The most common CRT related acute toxicities were nausea, leucopenia and thrombocytopenia. Grade 3 toxicity was detected in 13 (\%28.8) cases. No grade 4 toxicity was occurred. 
Table 1. Patients' and Tumor Characterics

\begin{tabular}{llrr}
\hline Characteristic & & $\mathrm{n}(\%)$ \\
\hline Median age & & $43(14-79)$ \\
Gender & Male & 32 & $(71.1)$ \\
& Female & 13 & $(28.9)$ \\
Stage & 1 & 3 & $(6.7)$ \\
& 2 & 9 & $(20)$ \\
& 3 & 18 & $(40)$ \\
& 4 & 15 & $(33.3)$ \\
Pathologic type & WHO IIb & 34 & $(75.5)$ \\
& Other & 11 & $(24.5)$ \\
Chemotherapy & Neoadjuvant & 15 & $(33.3)$ \\
& Neoadjuvant plus concurrent & 38 & $(84.4)$ \\
\hline
\end{tabular}

Table 2. Acute Toxicity

\begin{tabular}{lcccc}
\hline Toxicity & $\begin{array}{c}\text { Grade I } \\
\mathrm{n}(\%)\end{array}$ & $\begin{array}{c}\text { Grade II } \\
\mathrm{n}(\%)\end{array}$ & $\begin{array}{c}\text { Grade III } \\
\mathrm{n}(\%)\end{array}$ & $\begin{array}{c}\text { Grade IV } \\
\mathrm{n}(\%)\end{array}$ \\
\hline Mucositis & $17(37)$ & $25(55.6)$ & -6.7 & 0 \\
Skin reaction & $21(46.7)$ & $24(53.3)$ & 0 & 0 \\
Dysphagia & $10(22.2)$ & $31(68.9)$ & $4(8.9)$ & 0 \\
Gastrointestinal & $30(66.6)$ & $12(26.6)$ & $3(6.6)$ & 0 \\
Hematologic & $6(13.3)$ & $13(28.9)$ & $1(2.2)$ & 0 \\
\hline
\end{tabular}

Acute toxicities by site and grade are detailed in Table 2 . During radiotherapy $\geq 10 \%$ weight loss was observed in 15 patients. Mean weight loss was $9 \%$. Six cases were required adaptive plan due to tumor shrinkage or weight loss during treatment. None of the patients required the insertion of PEG for nutritional support. Radiation therapy was completed without interruption in all patients.

\section{Discussion}

NPC is rare and has distinct geographic and racial property. Local control in T1 and T2 NPC is at least 90\% and $85-90 \%$ with RT (Xiao et al., 2009). In the studies which compared RT alone and RT with chemotherapy, progression-free and overall survival in the concomitant applications have shown significant advantages over RT alone and has been adopted as the standard treatment for locally advanced NPC (Langendijk et al., 2004; Lee et al., 2009).

Treatment planning for NPC is a great challenge due to complex anatomical localization of the tumor and the surrounding critical structures. Sparing of normal tissues from severe toxicities during NPC radiotherapy is important because of large field and higher doses. IMRT is an optimal radiation method for NPC, due to ability of maximizing radiation dose to the target while sparing surrounding tissues. Encouraging results of IMRT in NPC have been reported (Kuang et al., 2012; Su et al., 2012).

Acute toxicity is important due to, it may lead interruption during treatment and predisposing to late side effects. Mucositis is the major factor affecting acute toxicity which causes deterioration of the patients' diet. Sun et al. observed acute mucositis in 868 NPC patients treated by IMRT. Incidences of grade 2 and 3 acute mucositis were, $49.7 \%$ and $21.5 \%$ for the patients who did not receive concomitant $\mathrm{CT}$ and $40.8 \%$, and $43.9 \%$; for patients who received CT respectively (Sun et al., 2014). In a study included 198 stage 1-2b NPC patients treated with IMRT alone, the incidence of acute $\geq$ grade 3 mucositis and pharyngitis was $13.6 \%$ and $1.0 \%$, respectively (Su et al., 2012).

The incidence of grade 1 and $\geq$ grade 2 dermatitis have been reported as $82.7 \%$ and $14.9 \%$ respectively with IMRT in the literature (Yi et al., 2006; Wong et al., 2010). While grade 3 dermatitis was reported $12 \%$ with conventional RT, it was reduced to $4 \%$ with IMRT (Wang et al., 2013). In a retrospective study comparing IMRT and conformal radiotherapy acute radiation dermatitis was reduced with IMRTP (Kuang etal., 2012). Zhao et al. demonstrated that acute grade 3 dermatitis was $23.6 \%$ with SIB tecnique (Zhao et al., 2012). Kong et al implemented IMRT in 364 NPC patients with cisplatin-based CT for local-regionally advanced disease. Grade 0-2 dermatitis and mucositis occurred in $92.6 \%$ and $56.1 \%$ respectively. While grade 3 dermatitis and mucositis were seen in $7.4 \%$ and $44 \%$ patients, no grade 4 acute toxicities were observed (Kong et al., 2014). In a multicenter prospective study, 300 NPC patients received definitive IMRT with CT; while grade 0-2 mucositis and dermatitis developped in $66.7 \%$ and $96.0 \%$ of patients, $\geq$ grade 3 mucositis and dermatitis were detected in $33.3 \%$ and $4.0 \%$ of patients, respectively (Wang et al., 2013).

In the study included 175 patients treated with WFSIB ; the incidence of acute grade 3 or higher mucositis/ pharyngitis was $23.4 \%$ during the IMRT course (Wong et al., 2010). Lin et al. (2010) treated 382 NPC patients with IMRT and CT (12.5\%). Grade 3 mucositis, skin desquamation, and leucocytopenia, were developped in $27.5 \%, 4.6 \%$, and $5.9 \%$ patients, respectively. In the present study mucositis has been identified in all patients; including grade 2 in $25(55.6 \%)$ and grade 3 in $3(6.7 \%)$ patients and the earliest time for emergence of this complication has been second week of the treatment. Sucralfate, prostaglandins, antiinflammatory and antimicrobial agents have been used in the prophylaxis, symptomatic and palliative therapy of radiotherapy mucositis. As well, all patients developped acute dermatitis; grade 1 in $21(46.7 \%)$ and grade 2 in 24 $(53.3 \%)$ patients respectively.

Also, dysphagia is common and usually begins the second week of treatment, can take 3-4 weeks. Zhao et al. observed grade 2 dysphagia in $8.5 \%$ of 193 patients who treated by IMRT (Zhao et al., 2012). In the current study grade 2 or higher dysphagia was recorded in 35 $(77.8 \%)$ patients.

Kong et al. (2014) showed grade 3 anemia, leukopenia, and thrombocytopenia in $2.5 \%, 17.3 \%$ and $6.2 \%$ of the patients, respectively and $13.6 \%$ of the patients experienced grade 4 leukopenia. In the series of 193 NPC patients treated by IMRT with SIB technique, acute grade 3 neutropenia occurred in $2.4 \%$ patients (Zhao et al., 2012). In another study; among 58 patients who underwent SIB-IMRT for NPC, $6.9 \%$ of patients developped grade 3 leucopenia (Xiang et al., 2013). In Zhao et al. study $85.5 \%$ of patients received chemotherapy, grade 2 and grade 3 acute stomatitis were observed in $52.3 \%$ and in $2.1 \%$ of patients (Zhao et al., 2012). In the present study chemotherapy was administered to 38 patients $(84.4 \%)$ as part of their treatments. When we evaluate acute toxicities related to CRT; most common toxicities were vomiting and 
stomatitis, grade 2 in $26.6 \%$ patients and grade 3 in $6.6 \%$ patients. As a result, mean weight loss has been reported as $9 \%$ and 6 cases were required adaptive plan during treatment. Also, hematological toxicity occurred related to CRT. The worst was grade 3 neutropenia in $2.2 \%$ patients.

In our study the most frequently observed acute toxicity during IMRT was mainly grade 1 or grade 2 . As well, few significant acute toxicities were observed. In general the toxicity profile was acceptable. No patient needed gastrostomy during the course of radiotherapy. Nutritional and analgesic support is required throughout the treatment. All patients were able to complete the whole course of irradiation without treatment interruption.

In conclusion, the results of our study suggest that; IMRT is a safe and effective treatment modality, and well tolerated by patients in the treatment of nasopharyngeal carcinoma. No unexpected side effect was observed. In order to overcome observed side effects, regular followup and administration of supportive therapy are required.

\section{References}

Chen CY, Han F, Zhao C, et al (2008). Long-term results of 934 nasopharyngeal carcinoma treated with radiotherapy alone. Chin J Radiat Oncol, 17, 411-5.

Edge SB, Byrd DR, Compton CC, et al (2009). American Joint Committee on Cancer, American Cancer Society. AJCC Cancer Staging Manual, $7^{\text {th }}$ edn. Springer, Berlin Heidelberg New York.

Kong F-f, Ying H, Du C-R, et al (2014). Effectiveness and toxicities of intensity-modulated radiation therapy for patients with $\mathrm{t} 4$ nasopharyngeal carcinoma. PLOS ONE, 9,91362.

Kuang WL, Zhou Q, Shen LF (2012). Outcomes and prognostic factors of conformal radiotherapy versus intensitymodulated radiotherapy fornasopharyngeal carcinoma. Clin Transl Oncol, 14, 783-90.

Langendijk JA, Leemans CR, Buter J, Berkhof J, Slotman BJ (2004). The additional value of chemotherapy to radiotherapy in locally advanced nasopharyngeal carcinoma: a meta-analysis of the published literature. J Clin Oncol, 22, 4604-12.

Lee AW, Lau WH, Tung SY, et al (2005). Preliminary results of a randomized study on therapeutic gain by concurrent chemotherapy for regionally-advanced nasopharyngeal carcinoma: NPC-9901 trial by the Hong Kong nasopharyngeal cancer study group. J Clin Oncol, 23, 6966-75.

Lee AW, Sze WM, Au JS, et al (2005). Treatment results for nasopharyngeal carcinoma in the modern era: the Hong Kong experience. Int J Radiat Oncol Biol Phys, 61, 1107-16.

Lee N, Harris J, Garden AS, et al (2009). Intensity modulated radiation therapy with or without chemotherapy for nasopharyngeal carcinoma: radiation therapy oncology group phase II trial 0225. J Clin Oncol, 27, 3684-90.

Lee N, Xia P, Quivery JM, et al (2002). Intensity-modulated radiotherapy in the treatment of nasopharyngeal carcinoma: An update of the UCSF experience. Int J Radiat Oncol Biol Phys, 53, 12-22.

Leung TW, Tung SY, Sze WK, et al (2005). Treatment results of 1070 patients with nasopharyngeal carcinoma: an analysis of survival and failure patterns. Head Neck, 27, 555-65.

Li-Na Zhao, Bin Zhou, Mei Shi, et al (2012). Clinical outcome for nasopharyngeal carcinoma with predominantly WHO II histology treated with intensity-modulated radiation therapy in non-endemic region of China. Oral Oncol, 48, 864-9.
Lin S, Lu JJ, Han L, Chen Q, Pan J (2010). Sequential chemotherapy and intensity-modulated radiation therapy in the management of locoregionally advanced nasopharyngeal carcinoma: experience of 370 consecutive cases. BMC Cancer, 10, 39.

Phua Chee Ee V, Tan BS, Tan AL, et al (2013). Dose planning study of target volume coverage with intensity-modulated radiotherapy for nasopharyngeal carcinoma: Penang General Hospital experience. Asian Pac J Cancer Prev, 14, 2243-8.

Saleh-Ebrahimi et al (2013). Intensity modulated radiotherapy (IMRT) combined with concurrent but not adjuvant chemotherapy in primary nasopharyngeal cancer - a retrospective single center analysis. Radiation Oncology, $\mathbf{8}, 20$.

Su SF, Han F, Zhao C, et al (2012). Long-term outcomes of early-stage nasopharyngeal carcinoma patients treated with intensity-modulated radiotherapy alone. Int J Radiat Oncol Biol Phys, 82, 327-33.

Sun X, Su S, Chen C, et al (2014). Long-term outcomes of intensity-modulated radiotherapy for 868 patients with nasopharyngeal carcinoma: An analysis of survival and treatment toxicities. Radiother Oncol, 110, 398-403.

Wang R, Wu F, Lu H, et al (2013). Definitive intensity-modulated radiation therapy for nasopharyngeal carcinoma: long-term outcome of a multicenter prospective study. J Cancer Res Clin Oncol, 139, 139-45.

Wong FC, Ng AW, Lee VH, et al (2010). Whole-field simultaneous integrated-boost intensity-modulated radiotherapy for patients with nasopharyngeal carcinoma. Int J Radiat Oncol Biol Phys, 76, 138-45.

Xiang L, Wang Y, Xu BQ, et al (2013). Preliminary results of a phase I/II study of simultaneous boost irradiation radiotherapy for locally advanced nasopharyngeal carcinoma. Asian Pac $J$ Cancer Prev, 14, 7569-76.

Xiao WW, Han F, Lu TX, et al (2009). Chen CY, Huang Y, Zhao C. Treatment outcomes after radiotherapy alone for patients with early-stage nasopharyngeal carcinoma. Int $J$ Radiat Oncol Biol Phys, 74, 1070-6.

Yi JL, Gao L, Huang XD, et al (2006). Nasopharyngeal carcinoma treated by radical radiotherapy alone: ten-year experience of a single institution. Int J Radiat Oncol Biol Phys, 65, 161-8. 